www.jmscr.igmpublication.org

Impact Factor 5.84

Index Copernicus Value: 83.27

ISSN (e)-2347-176x ISSN (p) 2455-0450

crossref DOI:_https://dx.doi.org/10.18535/jmscr/v5i6.12

Journal Of Medical Science And Clinical Research

Original Article

\title{
Impact of Oral Health Status, Oral Health Related Quality of Life and Social Determinants of Health on the Academic performance of 12 and 15 Year Old Children in Bangalore - A Descriptive study
}

\author{
Authors \\ Dr Deesha Kumari ${ }^{1}$, Dr Arkalgudu Govindraju Harikiran², Dr Jyotsna Srinagesh ${ }^{3}$ \\ ${ }^{1}$ Assistant Professor, AB Shetty Memorial Institute of Dental Sciences, Deralakatte \\ ${ }^{2}$ Prof and Head, Department of Public Health Dentistry, DAPM RV Dental College, $24^{\text {th }}$ main, JP Nagar $1^{\text {st }}$ \\ Phase, Bangalore, Karnataka \\ ${ }^{3}$ Reader, Department of Public Health Dentistry, DAPM RV Dental College, $24^{\text {th }}$ main, JP Nagar $1{ }^{\text {st }}$ Phase, \\ Bangalore, Karnataka \\ Corresponding Author
}

Dr Deesha Kumari

DAPM RV Dental College, $24^{\text {th }}$ main, JP Nagar $1^{\text {st }}$ Phase, Bangalore- 560078, Karnataka

Email:drdeesha.k@gmail.com, Phone No: +919611758152

\begin{abstract}
Oral health problems in children have a detrimental impact on their daily performance and quality of life causing missed school days and poor academic performance. The present study was conducted to measure the impact of Oral Health Status, Oral Health Related Quality Of Life and Social Determinants of Health on the Academic Performance of 12 and 15 year old school children in Bangalore, Karnataka. A crosssectional study employing a multi-stage cluster random sampling strategy was conducted among 860 children aged 12 and 15 years. The assessment was carried out in 3 sections including oral health status examination by calibrated examiners, Oral Health Related Quality of life assessment using COHIP questionnaire for children and Social Determinants of Health assessment by using a parental questionnaire. The children's previous year academic grades were obtained from the school authorities. Mother's education and missed school days due to oral problems were significantly associated with the Academic Performance of both 12 and 15 year old children. The ordinal regression model, established that with every 1 day decrease in the missed school days due to oral problems or 1 higher education qualification of mother, the Academic Performance of children increased to the next higher grade. The study revealed that oral Health Status independent of the social determinants of health influenced the Academic Performance of children. This initiates the need to influence policy makers and stakeholders to inculcate solitary or integrated oral health interventions in schools, thus aiming at effective oral health promotion.

Keywords: "Academic Performance", "Bangalore", "Oral Health Related Quality of Life", "Oral Health Status ", "Social determinants of Health" "School Children".
\end{abstract}

\section{INTRODUCTION}

Oral diseases, being universal in nature affect all individuals globally, irrespective of age or gender.
Despite huge oral health promotion activities, oral problems still persist, particularly among the under-privileged groups in developed and 
developing countries ${ }^{1}$. In India, a National Oral health Survey in 2002-03, revealed a mean DMFT of 1.7 and 2.3 among 12 and 15 year old children respectively with a twofold increase in adults ${ }^{2}$.

Poor oral health in children affects their mental, emotional and psychological well-being, thus hampering their growth and quality of life. In addition, poor oral health can root to distraction during lectures or missed school days, thus impeding their academic performance. Children with oral problems lose more than 51 million school hours annually and are 12 times more likely to have restricted-activity days than their counterparts ${ }^{3}$.

However, academic performance of children is also influenced by various other factors like their learning capability, academic training quality in schools, school environment, quality of life and socio-demographic determinants. Thus, a comprehensive assessment of oral health and other factors on academic performance is essential.

Oral health can be assessed through a normative approach by analyzing the oral health status or quality of life measures which incorporates concepts of functional, psychological and social well-being of the individual. Using Oral Health Related Quality Of Life (OHRQoL) measures alongside traditional clinical Oral Health Status assessment, a comprehensive outlook of the oral diseases impact on the several dimensions of subjective wellbeing becomes possible. ${ }^{4}$

Limited research globally have linked the influence of Oral Health Status, OHRQoL and Social Determinants on Academic Performance of children $^{3,5-7}$, whereas in India, studies persist which examine the influence of either oral health on academic performance or social determinants of health on academic performance, thus not signifying the independent influence of the variables on academic performance ${ }^{8}$. In India, the academic performance is an upshot of variables like social determinants (income, socio-economic status), overall health status and quality of life, which determines their success in future.

Hence the present descriptive study aims to assess the independent influence of Oral Health Status,
OHRQoL and Social Determinants on the Academic Performance of 12 and 15 year old school children in Bangalore.

\section{METHODOLOGY}

A descriptive study was conducted in Bangalore, Karnataka in 2014-2015 with a sample of 860 school children aged 12 and 15 years. Ethical clearance was obtained from the Institutional Review Board [IRB N0: 045/Vol-1/2013).

\section{Selection of Study Participants}

A multi-stage cluster random sampling was followed (Figure I). Children from government schools which had both higher primary and secondary school administration, aged 12 years (studying in standard $6^{\text {th }}$ or $7^{\text {th }}$ and born between $1^{\text {st }}$ August 2002 to $31^{\text {st } J u l y ~ 2003) ~ a n d ~} 15$ years (studying in standard $9^{\text {th }}$ or $10^{\text {th }}$ and born between $1^{\text {st }}$ August 1999 to $31^{\text {st } J u l y ~ 2000), ~ p r o v i d i n g ~}$ written informed consent and present during the assessment were included. The sample size was estimated using the formula $\mathrm{N}=\underline{\mathrm{Z}^{2} \mathrm{x}} \mathrm{PQ} \mathrm{XD} / \Delta^{2}$ where $Z=1.96$ for $95 \%$ Confidence level, $P$ $=$ Proportion of children aged 12 and 15 year old with good oral health $(0.5)^{9}, \mathrm{Q}=1-\mathrm{P}, \Delta=0.05(5 \%$ margin of error) and $\mathrm{D}=$ Design effect of $2^{10}$.

\section{Development and validation of the tools}

The study was conducted using a translated version of the Child oral Health Impact Profile -19 item generic questionnaire ${ }^{11}$ for the OHRQoL assessment (5 domains namely Oral Health-well being, Functional well-being, Social/ Emotional well-being, Social environment and Self image) and 9 item self-administered questionnaire on Social Determinants of Health. Both questionaires were in Kannada language. A bilingual professor translated the questionnaire from English to Kannada, while another independent bilingual professor translated the Kannada questionnaire back to English language. The differences were noted, and sorted out by involving both the translators. The Oral Health Status Assessment was conducted using an Oral health assessment form comprising of 2 indices: Dentition Status and Treatment Needs Index and Gingival index. All the tools were validated for 
face and content validity by a team of 6 Public Health Dentists

\section{Data Collection Phase}

Oral health examination by conducted by 03 calibrated examiners following the WHO guidelines, during which the study participants were identified. The OHRQoL questionnaire was later administered to the children. Consecutively, they were given the Social Determinants of Health questionnaire and instructed to get them completed by their parents and carry it to school the next day. Schools in Karnataka, during 1 academic year consist of 2 semesters with 2 formative assessments and 1 summative assessment per semester. Considering all 6 assessments, a final grade is assigned to the student. The final grade of the students from the previous academic year was obtained from the school authorities using a data extraction form.

\section{Statistical Analysis}

The data was analysed using SPSS version 16. The Social determinants and academic performance details were expressed in as number and percentage. The age-wise comparison of Oral Health Status, OHRQoL and Academic Performance was assessed using Man-Whitney U test. The gender-wise comparison of the OHRQoL and Academic Performance was assessed using chi-square test. The association of various independent variables on the academic performance was assessed using ordinal regression model. $\mathrm{p}<0.05$ was considered significant.

\section{RESULTS}

Among 860 school children, the data of 731 $(85 \%)$ children were analysed according to the per protocol analysis $^{12}$ and $15 \%$ data were excluded from the final analysis due to incomplete questionnaires returned and failure to obtain the questionnaires even after repeated reminders.

\section{Social determinants of the participants}

The distribution of participants was as follows: 12 year old - 289 (39.5\%), 15 year old- $442(60.5 \%)$, females- 326 (44.6) and males - 405 (55.4\%). The sample comprised of a preponderance of Hindus $(85 \%)$, with majority of the parents being illiterate
(30\%) pursuing unskilled occupation in the private firm (54-56\%). Although $26.81 \%$ individuals were unaware of their monthly family income, a majority of $26.95 \%$ individuals obtained an income of 9,249-13,873 (Table 1).

\section{Oral health status}

The overall DMFT score was marginally higher among the 15 year old $(0.99)$ than the 12 year old (0.98), however the age-wise analysis revealed no significant difference in the oral health status (Table 2). The gender wise analysis revealed a significant higher filled score in males as compared to females among 15 year old (Table 3).

\section{Oral Health Related Quality Of Life of children}

OHRQoL was assessed on a five point likert scale (Almost all time, fairly often, sometimes, almost never, never). A significant age-wise [(15 year 60.14, 12 year-53.92)] and gender-wise difference in the OHRQoL scores was observed indicating a better OHRQoL among 15 year old (Table 4) and females aged 15 years (Table 5).

\section{Academic performance of school children}

The academic performance of children was categorized grade wise as $\mathrm{A}^{+}, \mathrm{A}, \mathrm{B}^{+}, \mathrm{B}$ and $\mathrm{C}$.

Majority of the students obtained " $A$ " grade with a significantly higher academic grade amongst the 15 year old (Table 6) but no significant gender wise difference.

\section{Influence of various variables on the Academic Performance of children}

Among the various factors, the number of missed school days due to oral problems determining the OHRQoL (Table 7) and mothers education among the social determinants (Table 9), was significantly associated with the Academic Performance of children i.e. with every 1 day decrease in the missed school days or 1 higher educational qualification of the mother, the Academic Performance would significantly increase by 1 grade. 
Table I: Social determinants affecting the health of the study participants

\begin{tabular}{|c|c|c|c|}
\hline Sl. & \multicolumn{2}{|l|}{ Characteristic } & $\mathrm{n}(\%)$ \\
\hline & \multicolumn{3}{|c|}{ Religion wise Distribution of participants } \\
\hline & \multirow[t]{2}{*}{ Hindu } & Male & $285(38.99 \%)$ \\
\hline & & Female & $339(46.37 \%)$ \\
\hline & \multirow[t]{2}{*}{ Muslim } & Male & $8(1.1 \%)$ \\
\hline & & Female & $10(1.36 \%)$ \\
\hline & \multirow[t]{2}{*}{ Christian } & Male & $33(4.51 \%)$ \\
\hline & & Female & $56(7.66 \%)$ \\
\hline & \multicolumn{2}{|l|}{ Total } & $731(100.0)$ \\
\hline & Parents educational qualification & Mother & Father \\
\hline & Professional or honors & 0 & $1(0.13)$ \\
\hline & Graduate or post graduate & $1(0.13)$ & $3(0.41)$ \\
\hline & Post high school diploma & $22(3.01)$ & $39(5.34)$ \\
\hline & High school certificate & $211(28.86)$ & $195(26.68)$ \\
\hline & Middle school certificate & $143(19.56)$ & $128(17.51)$ \\
\hline & Primary school or literate & $130(17.78)$ & $148(20.25)$ \\
\hline & Illiterate & $224(30.64)$ & $217(29.69)$ \\
\hline & Total & $731(100.0)$ & $731(100.0)$ \\
\hline & Parents occupational status & Mother & Father \\
\hline & Professional & 0 & $3(0.41)$ \\
\hline & Semiprofessional & $2(0.27)$ & $3(0.41)$ \\
\hline & Clerical, shop owner, farmer & $198(27.09)$ & $133(18.19)$ \\
\hline & Skilled worker & $14(1.92)$ & $23(3.15)$ \\
\hline & Semi - skilled worker & $64(8.76)$ & $85(11.62)$ \\
\hline & Unskilled & $396(54.17)$ & $410(56.09)$ \\
\hline & Unemployed & $157(21.48)$ & $74(10.12)$ \\
\hline & Total & $731(100.00)$ & $731(100.00)$ \\
\hline
\end{tabular}

Table II: Comparison of Oral Health Status indicators between the 2 age groups

\begin{tabular}{|c|c|c|c|c|c|c|c|c|c|}
\hline Sl & Parameter & Age Group & $\mathrm{N}$ & Mean & Std Dev & SE of Mean & MD & $\mathrm{Z}$ & P-Value \\
\hline & \multirow{2}{*}{ DMFT Score } & $12 \mathrm{yrs}$ & 289 & 0.98 & 1.48 & 0.087 & \multirow{2}{*}{-0.004} & \multirow{2}{*}{-0.597} & \multirow{2}{*}{0.55} \\
\hline & & $15 \mathrm{yrs}$ & 442 & 0.99 & 1.61 & 0.076 & & & \\
\hline & \multirow{2}{*}{ Decay Score } & $12 \mathrm{yrs}$ & 289 & 0.88 & 1.39 & 0.082 & \multirow{2}{*}{0.006} & \multirow{2}{*}{-0.594} & \multirow{2}{*}{0.55} \\
\hline & & $15 \mathrm{yrs}$ & 442 & 0.87 & 1.47 & 0.070 & & & \\
\hline & \multirow{2}{*}{ Missing Score } & $12 \mathrm{yrs}$ & 289 & 0.06 & 0.32 & 0.019 & \multirow{2}{*}{0.001} & \multirow{2}{*}{0.918} & \multirow{2}{*}{0.92} \\
\hline & & 15 yrs & 442 & 0.05 & 0.32 & 0.015 & & & \\
\hline & \multirow{2}{*}{ Filled Score } & 12 yrs & 289 & 0.05 & 0.33 & 0.020 & \multirow{2}{*}{0.007} & \multirow{2}{*}{0.619} & \multirow{2}{*}{0.62} \\
\hline & & $15 \mathrm{yrs}$ & 442 & 0.05 & 0.24 & 0.011 & & & \\
\hline & \multirow{2}{*}{ GI } & $12 \mathrm{yrs}$ & 289 & 1.34 & 1.93 & 0.114 & \multirow{2}{*}{0.108} & \multirow{2}{*}{-0.582} & \multirow{2}{*}{0.56} \\
\hline & & $15 \mathrm{yrs}$ & 442 & 1.23 & 1.94 & 0.092 & & & \\
\hline
\end{tabular}

Table III: Gender wise Comparison of oral health indicators in 15 year old children

\begin{tabular}{|c|c|c|c|c|c|c|c|c|c|}
\hline S1 & Parameter & Age Group & $\mathrm{N}$ & Mean & $\begin{array}{l}\text { Std } \\
\text { Dev }\end{array}$ & $\begin{array}{l}\text { SE } \\
\text { Mean }\end{array}$ & $\mathrm{MD}$ & $\mathrm{Z}$ & P-Value \\
\hline & \multirow{2}{*}{ DMFT Score } & Male & 201 & 0.89 & 1.48 & 0.105 & \multirow{2}{*}{-0.176} & \multirow{2}{*}{-0.787} & \multirow{2}{*}{0.43} \\
\hline & & Female & 241 & 1.07 & 1.70 & 0.110 & & & \\
\hline & \multirow{2}{*}{ Decay Score } & Male & 201 & 0.84 & 1.41 & 0.099 & \multirow{2}{*}{-0.060} & \multirow{2}{*}{-0.025} & \multirow{2}{*}{0.98} \\
\hline & & Female & 241 & 1.90 & 1.53 & 0.098 & & & \\
\hline & \multirow{2}{*}{ Missing Score } & Male & 201 & 0.03 & 0.21 & 0.015 & \multirow{2}{*}{-0.036} & \multirow{2}{*}{-0.676} & \multirow{2}{*}{0.49} \\
\hline & & Female & 241 & 0.07 & 0.39 & 0.025 & & & \\
\hline & \multirow{2}{*}{ Filled Score } & Male & 201 & 0.12 & 0.12 & 0.009 & \multirow{2}{*}{-0.056} & \multirow{2}{*}{-2.357} & \multirow{2}{*}{$0.018^{*}$} \\
\hline & & Female & 241 & 0.07 & 0.30 & 0.019 & & & \\
\hline & \multirow{3}{*}{ GI } & Male & 201 & 1.32 & 1.93 & 0.136 & \multirow{3}{*}{0.176} & \multirow{3}{*}{-1.521} & \multirow{3}{*}{0.12} \\
\hline & & Female & 241 & 1.15 & 1.95 & 0.125 & & & \\
\hline & & Female & 241 & 7.03 & 3.52 & 0.226 & & & \\
\hline
\end{tabular}

*denotes significant difference 
Table IV: Comparison of OHRQoL between the two age groups

\begin{tabular}{|l|c|c|c|c|c|}
\hline Age Group & $\mathrm{N}$ & Mean & Std Dev & $\mathrm{Z}$ & P-Value \\
\hline $12 \mathrm{yrs}$ & 289 & 53.92 & 13.14 & \multirow{2}{*}{-6.828} & $<0.000^{*}$ \\
\hline $15 \mathrm{yrs}$ & 442 & 60.14 & 12.53 & & \\
\hline
\end{tabular}

*denotes significant association

Table V: Gender wise Comparison of OHRQoL scores

\begin{tabular}{|c|c|c|c|c|c|c|}
\hline Age Group & Gender & $\mathrm{N}$ & Mean & Std Dev & Z & P-Value \\
\hline \multirow{2}{*}{$12 \mathrm{yrs}$} & Male & 125 & 53.14 & 13.06 & \multirow{2}{*}{-0.902} & \multirow{2}{*}{0.367} \\
\hline & Female & 164 & 54.52 & 13.21 & & \\
\hline \multirow{2}{*}{$15 \mathrm{yrs}$} & Male & 201 & 57.49 & 14.24 & \multirow{2}{*}{-3.454} & \multirow{2}{*}{$<0.001^{*}$} \\
\hline & Female & 241 & 62.34 & 10.42 & & \\
\hline
\end{tabular}

*denotes significant difference

Table VI: Age wise Academic Performance of children

\begin{tabular}{|c|c|c|c|c|c|c|}
\hline $\begin{array}{l}\text { Grades } \\
\text { obtained }\end{array}$ & Age group & $\mathrm{n}(\%)$ & Mean & Std Dev & $\mathrm{Z}$ & P-Value \\
\hline \multirow{5}{*}{$\begin{array}{l}12 \text { years } \\
(\mathrm{n}=289)\end{array}$} & A+ & $70(24.2 \%)$ & \multirow{5}{*}{2.43} & \multirow{5}{*}{1.180} & \multirow{11}{*}{-2.704} & \multirow{11}{*}{$0.007^{*}$} \\
\hline & A & $104(36.0 \%)$ & & & & \\
\hline & $\mathrm{B}+$ & $52(18 \%)$ & & & & \\
\hline & B & $47(16.3 \%)$ & & & & \\
\hline & $\mathrm{C}$ & $16(5.5 \%)$ & & & & \\
\hline \multirow{5}{*}{$\begin{array}{l}15 \text { years } \\
\mathrm{N}=(442)\end{array}$} & $\mathrm{A}+$ & $79(17.9 \%)$ & \multirow{6}{*}{2.66} & \multirow{6}{*}{1.174} & & \\
\hline & A & $137(31 \%)$ & & & & \\
\hline & $\mathrm{B}+$ & $115(26 \%)$ & & & & \\
\hline & B & $79(17.9 \%)$ & & & & \\
\hline & $\mathrm{C}$ & $32(7.2 \%)$ & & & & \\
\hline \multicolumn{2}{|c|}{ Total } & 731 (100.0) & & & & \\
\hline
\end{tabular}

*denotes significant difference

Table VII: Influence of OHRQoL on the Academic Performance of children

\begin{tabular}{|c|c|c|c|c|c|c|c|c|}
\hline \multirow{2}{*}{ Sl } & \multirow{2}{*}{\multicolumn{2}{|c|}{ OHRQoL }} & \multirow{2}{*}{$\beta$} & \multirow{2}{*}{ SE of $\beta$} & \multirow{2}{*}{ Odds Ratio } & \multirow{2}{*}{ P-Value } & \multicolumn{2}{|c|}{$95 \%$ CI for $\beta$} \\
\hline & & & & & & & Lower & Upper \\
\hline & \multirow{2}{*}{ Pain } & $12 \mathrm{yr}$ & 0.179 & 0.106 & 1.196 & 0.091 & -0.029 & 0.387 \\
\hline & & $15 \mathrm{yr}$ & -0.172 & 0.096 & 0.842 & 0.074 & -0.361 & 0.017 \\
\hline & \multirow{2}{*}{ Discoloration } & $12 \mathrm{yr}$ & 0.068 & 0.087 & 1.071 & 0.430 & -0.101 & 0.238 \\
\hline & & $15 \mathrm{yr}$ & -0.036 & 0.072 & 0.965 & 0.616 & -0.177 & 0.105 \\
\hline & \multirow{2}{*}{ Crooked/spacing } & $12 \mathrm{yr}$ & 0.086 & 0.066 & 1.090 & 0.191 & -0.043 & 0.214 \\
\hline & & $15 \mathrm{yr}$ & -0.046 & 0.064 & 0.955 & 0.468 & -0.171 & 0.078 \\
\hline & \multirow{2}{*}{ Halitosis } & $12 \mathrm{yr}$ & 0.088 & 0.095 & 1.092 & 0.353 & -0.098 & 0.274 \\
\hline & & $15 \mathrm{yr}$ & -0.107 & 0.104 & 0.899 & 0.305 & -0.311 & 0.097 \\
\hline & \multirow{2}{*}{ Bleeding gums } & $12 \mathrm{yr}$ & 0.130 & 0.089 & 1.139 & 0.145 & -0.045 & 0.306 \\
\hline & & $15 \mathrm{yr}$ & -0.016 & 0.084 & 0.984 & 0.850 & -0.181 & 0.149 \\
\hline & \multirow{2}{*}{ Eating problem } & $12 \mathrm{yr}$ & -0.018 & 0.106 & 0.982 & 0.865 & -0.225 & 0.189 \\
\hline & & $15 \mathrm{yr}$ & -0.049 & 0.093 & 0.952 & 0.595 & -0.232 & 0.133 \\
\hline & \multirow{2}{*}{ Disturbed Sleep } & $12 \mathrm{yr}$ & -0.033 & 0.119 & 0.968 & 0.783 & -0.266 & 0.200 \\
\hline & & $15 \mathrm{yr}$ & -0.082 & 0.108 & 0.921 & 0.448 & -0.294 & 0.130 \\
\hline & \multirow{2}{*}{ Word pronunciation } & $12 \mathrm{yr}$ & -0.103 & 0.100 & 0.903 & 0.305 & -0.298 & 0.093 \\
\hline & & $15 \mathrm{yr}$ & 0.021 & 0.092 & 1.021 & 0.819 & -0.159 & 0.202 \\
\hline & Teeth clean & $12 \mathrm{yr}$ & 0.051 & 0.108 & 1.052 & 0.635 & -0.160 & 0.263 \\
\hline
\end{tabular}




\begin{tabular}{|c|c|c|c|c|c|c|c|c|}
\hline $\mathrm{S} 1$ & \multicolumn{2}{|l|}{ OHRQoL } & \multirow{2}{*}{$\frac{\beta}{0.118}$} & \multirow{2}{*}{$\frac{\text { SE of } \beta}{0.110}$} & \multirow{2}{*}{$\begin{array}{c}\text { Odds Ratio } \\
1.125\end{array}$} & \multirow{2}{*}{$\frac{\text { P-Value }}{0.282}$} & \multicolumn{2}{|c|}{$95 \%$ CI for $\beta$} \\
\hline & & $15 \mathrm{yr}$ & & & & & -0.097 & 0.333 \\
\hline & \multirow{2}{*}{ Sad feeling } & $12 \mathrm{yr}$ & -0.046 & 0.108 & 0.955 & 0.674 & -0.258 & 0.167 \\
\hline & & $15 \mathrm{yr}$ & 0.160 & 0.102 & 1.173 & 0.116 & -0.039 & 0.359 \\
\hline & \multirow{2}{*}{ Tensed } & $12 \mathrm{yr}$ & -0.241 & 0.105 & 0.786 & 0.022 & -0.447 & -0.035 \\
\hline & & $15 \mathrm{yr}$ & 0.007 & 0.106 & 1.007 & 0.944 & -0.201 & 0.216 \\
\hline & \multirow{2}{*}{$\begin{array}{l}\text { Refrainment while } \\
\text { speaking }\end{array}$} & $12 \mathrm{yr}$ & 0.091 & 0.113 & 1.095 & 0.422 & -0.131 & 0.313 \\
\hline & & $15 \mathrm{yr}$ & 0.068 & 0.116 & 1.071 & 0.556 & -0.160 & 0.296 \\
\hline & \multirow{2}{*}{ Been bullied } & $12 \mathrm{yr}$ & -0.076 & 0.116 & 0.926 & 0.508 & -0.303 & 0.150 \\
\hline & & $15 \mathrm{yr}$ & 0.123 & 0.115 & 1.131 & 0.287 & -0.103 & 0.349 \\
\hline & \multirow{2}{*}{ Felt ugly } & $12 \mathrm{yr}$ & 0.029 & 0.110 & 1.029 & 0.794 & -0.186 & 0.243 \\
\hline & & $15 \mathrm{yr}$ & 0.081 & 0.106 & 1.084 & 0.446 & -0.127 & 0.289 \\
\hline & \multirow{2}{*}{$\begin{array}{l}\text { Perturbed by others } \\
\text { thought }\end{array}$} & $12 \mathrm{yr}$ & -0.018 & 0.112 & 0.982 & 0.873 & -0.238 & 0.202 \\
\hline & & $15 \mathrm{yr}$ & -0.164 & 0.110 & 0.849 & 0.138 & -0.380 & 0.053 \\
\hline & \multirow{2}{*}{ Missed school } & $12 \mathrm{yr}$ & -0.356 & 0.120 & 0.700 & $0.003^{*}$ & -0.592 & -0.121 \\
\hline & & $15 \mathrm{yr}$ & -0.032 & 0.120 & 0.969 & $0.012^{*}$ & -0.467 & -0.114 \\
\hline & \multirow{2}{*}{ Talk aloud } & $12 \mathrm{yr}$ & 0.050 & 0.113 & 1.052 & 0.656 & -0.171 & 0.272 \\
\hline & & $15 \mathrm{yr}$ & -0.260 & 0.124 & 0.771 & 0.136 & -0.503 & -0.017 \\
\hline & \multirow{2}{*}{ Self confidence } & $12 \mathrm{yr}$ & -0.134 & 0.074 & 0.875 & 0.069 & -0.278 & 0.010 \\
\hline & & $15 \mathrm{yr}$ & 0.026 & 0.064 & 1.027 & 0.683 & -0.100 & 0.152 \\
\hline & \multirow{2}{*}{ Felt beautiful } & $12 \mathrm{yr}$ & 0.076 & 0.069 & 1.079 & 0.271 & -0.060 & 0.212 \\
\hline & & $15 \mathrm{yr}$ & 0.020 & 0.066 & 1.020 & 0.767 & -0.110 & 0.149 \\
\hline
\end{tabular}

*denotes a significantly influencing variable

Table VIII: Influence of Oral health status on the Academic Performance of children

\begin{tabular}{|c|c|c|c|c|c|c|c|c|}
\hline \multirow{2}{*}{$\mathrm{Sl}$} & \multirow{2}{*}{\multicolumn{2}{|c|}{ Oral health Status }} & \multirow{3}{*}{$\begin{array}{c}\beta \\
0.622\end{array}$} & \multirow{3}{*}{$\begin{array}{c}\text { SE of } \beta \\
1.866\end{array}$} & \multirow{3}{*}{$\begin{array}{l}\text { Odds } \\
\text { Ratio } \\
1.862 \\
\end{array}$} & \multirow{3}{*}{$\begin{array}{c}\text { P-Value } \\
0.739 \\
\end{array}$} & \multicolumn{2}{|c|}{$95 \%$ CI for $\beta$} \\
\hline & & & & & & & \multirow{2}{*}{$\begin{array}{l}\begin{array}{l}\text { Lower } \\
\text { Bound }\end{array} \\
-3.035 \\
\end{array}$} & \multirow{2}{*}{$\begin{array}{c}\begin{array}{c}\text { Upper } \\
\text { Bound }\end{array} \\
4.278 \\
\end{array}$} \\
\hline & \multirow{2}{*}{ Decay } & $12 \mathrm{yr}$ & & & & & & \\
\hline & & $15 \mathrm{yr}$ & -2.164 & 193.423 & 0.115 & 0.991 & -381.266 & 376.938 \\
\hline & \multirow{2}{*}{ Missing } & $12 \mathrm{yr}$ & 0.845 & 1.896 & 2.327 & 0.656 & -2.872 & 4.561 \\
\hline & & $15 \mathrm{yr}$ & -2.727 & 193.423 & 0.065 & 0.989 & -381.829 & 376.375 \\
\hline & \multirow{2}{*}{ Filled } & $12 \mathrm{yr}$ & 1.275 & 1.896 & 3.579 & 0.501 & -2.440 & 4.990 \\
\hline & & $15 \mathrm{yr}$ & -3.271 & 193.423 & 0.038 & 0.987 & -382.374 & 375.831 \\
\hline & \multirow{2}{*}{$\begin{array}{l}\text { DMFT } \\
\text { Score }\end{array}$} & $12 \mathrm{yr}$ & -0.281 & 1.865 & 0.755 & 0.880 & -3.936 & 3.374 \\
\hline & & $15 \mathrm{yr}$ & 2.979 & 193.423 & 19.664 & 0.988 & -376.123 & 382.080 \\
\hline & \multirow{2}{*}{ GI } & $12 \mathrm{yr}$ & 0.090 & 0.062 & 1.094 & 0.145 & -0.031 & 0.211 \\
\hline & & $15 \mathrm{yr}$ & 0.063 & 0.048 & 1.065 & 0.189 & -0.031 & 0.156 \\
\hline
\end{tabular}

*denotes a significantly influencing variable 
Table IX: Influence of Social determinants on the Academic Performance of children

\begin{tabular}{|c|c|c|c|c|c|c|c|c|}
\hline \multirow{2}{*}{$\mathrm{Sl}$} & \multirow{2}{*}{\multicolumn{2}{|c|}{ Social determinants }} & \multirow{3}{*}{$\begin{array}{c}\beta \\
0.176\end{array}$} & \multirow{3}{*}{$\begin{array}{c}\text { SE of } \beta \\
1.105\end{array}$} & \multirow{3}{*}{$\begin{array}{l}\text { Odds } \\
\text { Ratio } \\
1.185\end{array}$} & \multirow{3}{*}{$\begin{array}{c}\text { P-Value } \\
0.092\end{array}$} & \multicolumn{2}{|c|}{$95 \%$ CI for $\beta$} \\
\hline & & & & & & & \multirow{2}{*}{$\begin{array}{l}\text { Lower } \\
\text { Bound } \\
-0.025\end{array}$} & \multirow{2}{*}{$\begin{array}{c}\text { Upper } \\
\text { Bound } \\
0.384\end{array}$} \\
\hline & \multirow{2}{*}{ Age } & $12 \mathrm{yr}$ & & & & & & \\
\hline & & $15 \mathrm{yr}$ & 0.130 & 0.084 & 1.134 & 0.143 & -0.042 & 0.301 \\
\hline & \multirow{2}{*}{ Fathers education } & $12 \mathrm{yr}$ & -0.241 & 0.105 & 0.784 & 0.133 & -0.443 & -0.030 \\
\hline & & $15 \mathrm{yr}$ & -0.134 & 0.074 & 0.873 & 0.062 & -0.273 & 0.015 \\
\hline & \multirow{2}{*}{ Mothers education } & $12 \mathrm{yr}$ & 0.759 & 0.442 & 2.664 & $0.027^{*}$ & 0.117 & 1.890 \\
\hline & & $15 \mathrm{yr}$ & 0.887 & 0.553 & 2.038 & $0.007^{*}$ & 0.157 & 1.821 \\
\hline & \multirow{2}{*}{ Fathers occupation } & $12 \mathrm{yr}$ & 0.064 & 0.113 & 1.069 & 0.552 & -0.160 & 0.296 \\
\hline & & $15 \mathrm{yr}$ & 0.076 & 0.169 & 0.962 & 0.503 & -0.152 & 0.075 \\
\hline & \multirow{2}{*}{ Mothers occupation } & \multirow{2}{*}{$\begin{array}{l}12 \mathrm{yr} \\
15 \mathrm{yr}\end{array}$} & 0.081 & 0.106 & 1.082 & 0.441 & -0.123 & 0.285 \\
\hline & & & 0.038 & 0.151 & 1.039 & 0.800 & -0.257 & 0.334 \\
\hline & \multirow{2}{*}{ Family income } & $12 \mathrm{yr}$ & 0.808 & 0.514 & 2.240 & 0.110 & -0.205 & 1.814 \\
\hline & & $15 \mathrm{yr}$ & 0.040 & 0.064 & 1.035 & 0.762 & -0.112 & 0.151 \\
\hline
\end{tabular}

*denotes a significantly influencing variable

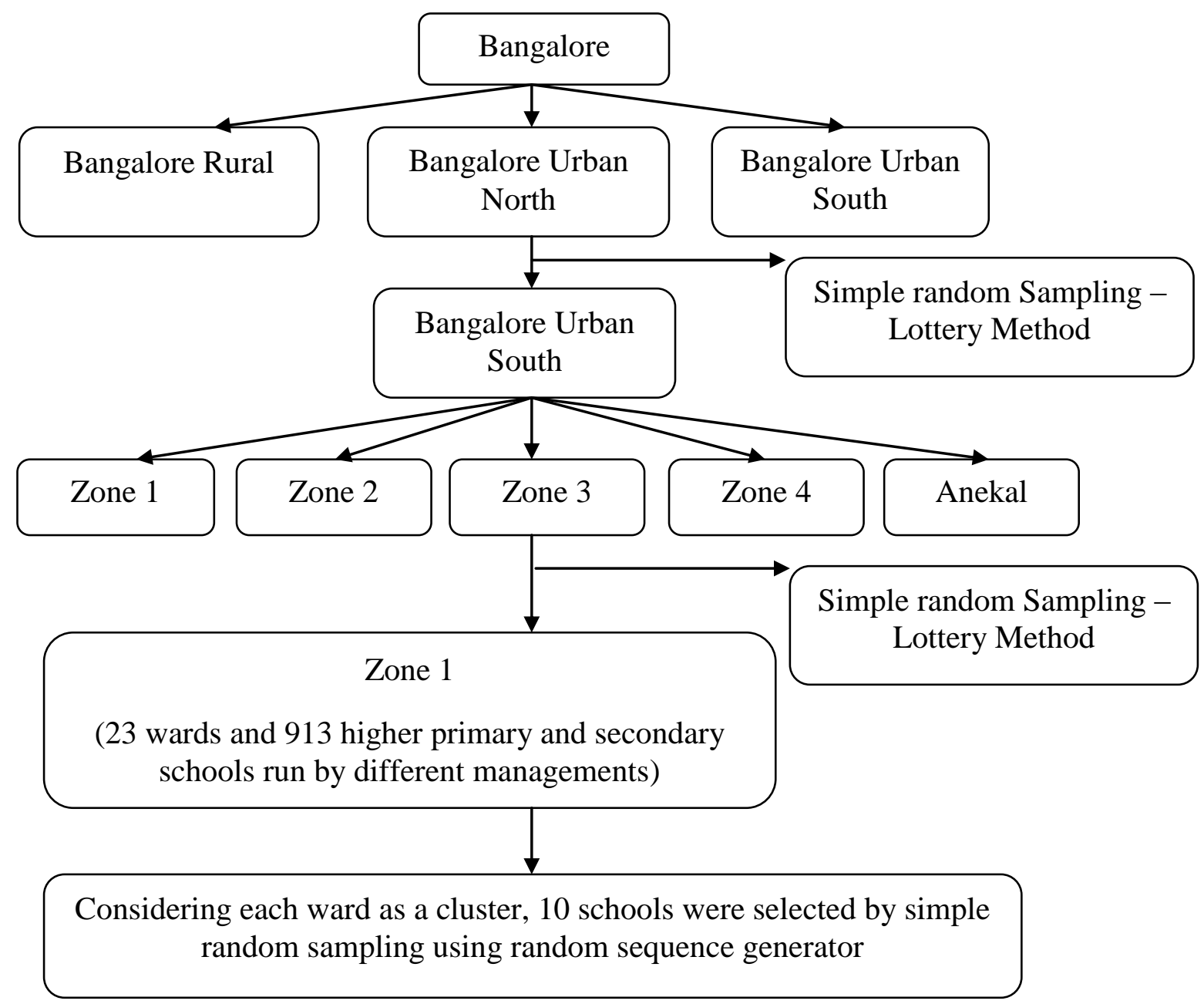

Figure I: Sampling Strategy employed in the study

\section{DISCUSSION}

Poor oral health can have a detrimental effect on children's daily life, hampering their quality of life and academic performance. Poor academic performance in children is one of the various factors responsible for school drop outs. Primary 
school level dropout was $9 \%$ for India as whole ranging from 1.8 to $15.5 \% .18 \%$ children dropped out of school before completing middle school ${ }^{13}$. The current study was conducted among 12 and 15 year old children as it is the recommended age for global monitoring of caries for international comparisons and monitoring of disease trends ${ }^{14}$.

The present study revealed that the OHRQoL of 15 year old children (60.14) was better than 12 year old (53.92). In addition, 15 year old females had a significantly better OHRQoL than males. Similar studies which assessed the factors associated with school absence in children instituted that the OHRQol was lower in 12 year old with no gender differences ${ }^{15}$. This may be because younger children have a lower threshold to pain or abnormal symptoms ${ }^{16}$. In the present study, the DMFT experience among 15 year old was marginally higher (0.99) than the 12 year old (0.98) with untreated decay being higher in 12 year old (0.88) than the 15 year old (0.87). But, a retrospective analysis of the caries experience in 5, 12 and 15 year old Indian children showed that the mean caries experience was higher among 15 year old (2.46) than the 12 year old (1.48). This may be due to the fact that the oral health status of the younger children is usually monitored by the parents and hence decreased caries experience.

The present study revealed that $36 \%$ of 12 year old children and $31 \%$ of 15 year old children scored an academic grade of " $\mathrm{A}$ " which is considered as "Excellent" according to the grading criteria. These findings are supported by the Sarva Siksha Abhiyan document of Karnataka, which affirms that Student Learning Study conducted by Educational Initiatives reveal that Karnataka clearly show an overall better performance than the national average ${ }^{17}$. The improved standards in educational delivery by introduction of formative assessment encourage students possessing lower grades to enhance their performance subsequently. Of the various social determinants of health, mothers' education seemed to have a significant impact on the academic performance of children. Studies assessing the influence of various factors on school performance showed that primary education of parents was significantly associated with academic performance ${ }^{3,7}$. Educated parents are more verbally responsive to their young children and tend to use teaching strategies that mimic formal instructional techniques, such as asking questions and offering feedback rather than issuing directives. However, the Oral Health Status assessment revealed no significant impact on academic performance in terms of dental caries experience (Table 8). The results are in contradiction with studies which proved that the caries experience in children was significantly associated with their academic performance ${ }^{6,20}$. This is because, in the present study caries experience was assessed using DMFT index which appraises both initial and advanced caries lesion similarly and thus the differentiation between the severe lesions which cause pain and discomfort in children leading to missed school days was not viable. The OHRQoL assessment revealed that, the number of missed school days due to oral problems was significantly associated with the Academic Performance of both 12 and 15 year old children. Similar results were obtained in a study assessing dental care with school absence $^{20}$. Oral problems cause pain, discomfort in children leading to missed school days which cause a disconnection in the knowledge attainment, thus hampering their Academic Performance.

The study has a good internal validity as the design and conduct of the present study has been conducted rigorously with minimal bias as per the STROBES Statement. To minimize the influence of variables, Ordinal regression was done by adjusting for the confounders. The study population may be considered similar to the target population in terms of their entry level requirements to the school i.e. minimum age limit - $6 \mathrm{yrs}$ and Socio-economic Status. Thus, the findings of the present study can be extrapolated to all higher primary and secondary school students in Bangalore.

However, the study has few limitations. The data was obtained through a questionnaire and so there may be an influence of social desirability bias. 
Even after repeated reminders to the students, personally and through the school teachers, all the parental questionnaires could not be obtained. Thus the Per Protocol analysis or Compliers only analysis was used which induces selection bias.

The findings suggest that the normative oral health status measurement is not comprehensive in its entity, thus resonating the need for incorporation of a more standardized measurement incorporating both the normative needs and OHRQoL assessment. In addition, through experience, the need for a more sensitive index to help identify missed school days due to oral problems is needed. Longitudinal longer followup study initiatives to study the influence of various factors on the Academic Performance of school children should be undertaken. Further research utilizing children of various age groups especially in rural areas, assessing the missed school days in children due to oral problems and analyzing its impact on the Academic Performance should also be initiated.

The present study reflects that academic performance of children is impacted by their oral health and social determinants of health. It can convince the curriculum developers / policy makers to recognize the importance of oral health integration into existing curricula by fortifying health promoting school strategies and implementing measures for training teachers and parents to detect the oral lesions at the early stage, thus targeting at oral health promotion.

Source of support: None

\section{REFERENCES}

1. WHO | What is the burden of oral disease? [Internet]. WHO. [cited 2015 Feb 17]. Available from: http://www.who.int/oral_health/disease_bu rden/global/en/

2. Bali R, Mathur V, Talwar R. National Oral health survey and Fluoride Mapping 20022003 [Internet]. 2004. Available from: http://www.dciindia.org.in/Download/Boo ks/NOHSBOOK.pdf
3. Paula JS de, Mialhe FL. Impact of oral health conditions on school performance and lost school days by children and adolescents: what are the actual pieces of evidence? Braz J Oral Sci. 2013 Sep;12(3):189-98.

4. Fotedar S, Sharma K rajeev, Fotedar V. Relationship between Oral Health Status and Oral Health Related Quality Of Life in Adults Attending H.P Government Dental College, Shimla, Himachal Pradesh- India. OHDM [Internet]. 2014 Sep;13(3). Available from: http://omicsonline.com/openaccess/relationship-between-oral-healthstatus-and-oral-health-related-quality-oflife-in-adults-attending-hp-governmentdental-college

5. Blumenshine SL, Vann WF, Gizlice Z, Lee JY. Children's school performance: impact of general and oral health. J Public Health Dent. 2008;68(2):82-7.

6. Piovesan C, Antunes JLF, Mendes FM, Guedes RS, Ardenghi TM. Influence of children's oral health-related quality of life on school performance and school absenteeism. J Public Health Dent. 2012;72(2):156-63.

7. Seirawan H, Faust S, Mulligan R. The Impact of Oral Health on the Academic Performance of Disadvantaged Children. Am J Public Health. 2012 Sep;102(9):1729-34.

8. Garg N, Anandakrishna L, Chandra P. Is there an Association between Oral Health Status and School Performance? A Preliminary Study. Int J Clin Pediatr Dent. 2012;5(2):132-5.

9. Census of India Website: Office of the Registrar General \& Census Commissioner, India [Internet]. [cited 2015 May 19]. Available from: http://censusindia.gov.in/

10. Kalton G, Brick J, Le T. Estimating components of design effects for use in sample design. 
11. Broder HL, Wilson-Genderson M, Sischo L. Reliability and validity testing for the Child Oral Health Impact Profile-Reduced (COHIP-SF 19). J Public Health Dent. 2012 Fall;72(4):302.

12. Matilde Sanchez M, Chen X. Choosing the analysis population in non-inferiority studies: per protocol or intent-to-treat. Stat Med. 2006 Apr 15;25(7):1169-81.

13. Gouda S, Sekher T. Factors Leading to School Dropouts in India: An Analysis of National Family Health Survey-3 Data. IOSR J Res Method Educ. 2014 Dec;4(6):75-83.

14. Pathfinder survey [Internet]. 2010 [cited 2015 Nov 1]. Available from: https://www.mah.se/CAPP/Methods-andIndices/for-Measurement-of-dentaldiseases/Extracts-from-WHO-Oral-HealthSurveys/Pathfinder-survey/

15. Krisdapong S, Prasertsom P, Rattanarangsima K, Sheiham A. School absence due to toothache associated with sociodemographic factors, dental caries status, and oral health-related quality of life in 12- and 15-year-old Thai children. J Public Health Dent. 2013 Sep 1;73(4):321-8.

16. Schmitz A, Vierhaus M. Pain tolerance in children and adolescents: Sex differences and psychosocial influences on pain threshold and endurance. Eur $\mathbf{J}$ Pain [Internet]. 2013 [cited 2017 Mar 25];17(1). Available from: https://www.researchgate.net/publication/2 27400655_Pain_tolerance_in_children_an d_adolescents_Sex_differences_and_psych osocial_influences_on_pain_threshold_and _endurance

17. sarva Siksha Abhiyan- 13th Joint review Mission [Internet]. 2011. Available from: http://ssa.nic.in/monitoringdocuments_old/jrm/13th\%20JRM/Revised $\%$ 20Karnataka.pdf
18. Ozmert EN, Yurdakök K, Soysal S, KulakKayikçi ME, Belgin E, Ozmert E, et al. Relationship between physical, environmental and sociodemographic factors and school performance in primary schoolchildren. J Trop Pediatr. 2005 Feb;51(1):25-32.

19. Muirhead V, Marcenes W. An ecological study of caries experience, school performance and material deprivation in 5year-old state primary school children. Community Dent Oral Epidemiol. 2004 Aug;32(4):265-70.

20. Pourat N, Nicholson G. Unaffordable dental care is linked to frequent school absences. Policy Brief UCLA Cent Health Policy Res. 2009 Nov;(PB2009-10):1-6. 